\title{
Many-body problem in Kaluza-Klein models with toroidal compactification
}

\author{
Alexey Chopovsky ${ }^{1,2, a}$, Maxim Eingorn ${ }^{2,3,4, b}$, Alexander Zhuk ${ }^{2, c}$ \\ ${ }^{1}$ Department of Theoretical Physics, Odessa National University, Dvoryanskaya st. 2, Odessa 65082, Ukraine \\ ${ }^{2}$ Astronomical Observatory, Odessa National University, Dvoryanskaya st. 2, Odessa 65082, Ukraine \\ ${ }^{3}$ Department of Theoretical and Experimental Nuclear Physics, Odessa National Polytechnic University, Shevchenko av. 1, Odessa 65044, Ukraine \\ ${ }^{4}$ CREST and NASA Research Centers, North Carolina Central University, Fayetteville st. 1801, Durham, NC 27707, USA
}

Received: 24 June 2013 / Accepted: 11 December 2013 / Published online: 23 January 2014

(C) The Author(s) 2014. This article is published with open access at Springerlink.com

\begin{abstract}
In this paper, we consider a system of gravitating bodies in Kaluza-Klein models with toroidal compactification of the extra dimensions. To simulate the astrophysical objects (e.g., our Sun and pulsars) with energy density much greater than the pressure, we assume that these bodies are pressureless in the external space, i.e., the space we inhabit. At the same time, they may have nonzero parameters $\omega_{(\bar{\alpha}-3)}(\bar{\alpha}=4, \ldots, D)$ in the equations of state in the extra dimensions. We construct the Lagrange function of this many-body system for any value of $\Sigma=\sum_{\bar{\alpha}} \omega_{(\bar{\alpha}-3)}$. Moreover, the gravitational tests (PPN parameters, perihelion and periastron advances) require a negligible deviation from the latent soliton value $\Sigma=-(D-3) / 2$. However, the presence of pressure/tension in the internal space results necessarily in the smearing of the gravitating masses over the internal space and in the absence of KK modes. This looks very unnatural from the point of view of quantum physics.
\end{abstract}

\section{Introduction}

The idea of multidimensionality of our Universe as required by the theories of unification of the fundamental interactions is one of the most breathtaking ideas of theoretical physics. It originates with the pioneering papers by Kaluza and Klein $[1,2]$, and now the most self-consistent modern theories of unification, such as superstrings, supergravity and M-theory, are constructed in spacetimes with extra dimensions (see, e.g., [3]). Different aspects of the idea of multidimensionality are intensively used in numerous modern articles.

\footnotetext{
a e-mail: a.chopovsky@yandex.ru

b e-mail: maxim.eingorn@gmail.com

c e-mail: ai.zhuk2@gmail.com
}

Therefore, it is important to find experimental evidence for the existence of the extra dimensions. For example, one of the aims of the Large Hadronic Collider consists in detecting of Kaluza-Klein (KK) particles which correspond to excitations of the internal spaces (see, e.g., [4]). Such excitations were investigated in a lot of articles (see, e.g., the classical papers [5-7]). Quite recently, KK particles were considered, e.g., in the papers $[8,9]$.

On the other hand, if we can show that the existence of the extra dimensions is contrary to observations, then these theories are prohibited.

Much work was done in this direction including the study of models with toroidal compactification. Obviously, any gravitational theory modified with respect to general relativity (GR) can result in some observable deviations from GR. A number of papers were devoted to the search of such deviations. For example, the nonrelativistic gravitational potentials in these theories can be different from the Newtonian potentials [10-16]. In principle, this difference can be experimentally observed [17]. The Parameterized Post-Newtonian (PPN) formalism is a powerful tool for the determination of gravitational theories consistent with experiments $[18,19]$.

The relation to particle physics is another important point of KK models. It was shown that multidimensional models can give a reasonable explanation of the hierarchy problem $[10,11]$. Next, it was indicated that such a framework can be embedded in string theory [20]. On the other hand, the interaction between KK states and ordinary matter can result in new observable channels of reactions [10,11,20-24].

In our previous papers $[19,25,26]$ devoted to KK models with toroidal compactification of the extra dimensions, we have shown that gravitating masses should have tension in the internal space to be in agreement with gravitational experiments in the Solar system. For example, black strings/branes with the parameter $\omega=-1 / 2$ in the equation of state in the 
internal space satisfy this condition. For this value of $\omega$, the variations of the internal space volume are absent [27]. In the dust-like case with $\omega=0$, such variations generate a fifth force, which leads to contradictions with the experimental data.

It is worth noting that black strings/branes generalize the known Schwarzschild solution to the multidimensional case (see, e.g., [28-31] and the corresponding literature therein). Obviously, any multidimensional theory should have such solutions, as they must correspond to the observed astrophysical objects. Black strings/branes have toroidal compactification of the internal spaces. This compactification type is the simplest among the possible ones. However, it makes sense to investigate such models because they may help to reveal new important properties for more physically reliable multidimensional models. The ADD model [10] presents a good example of it. Even if the authors use the localization of the Standard model fields on a brane, they explore the toroidal compactification of the internal space to get the relation between the multidimensional and four-dimensional gravitational constants [11]. That gives a possibility to solve the hierarchy problem and to introduce the notion of large extra dimensions. We will not use the brane approach for our model, remaining within the standard Kaluza-Klein theory. However, even in this case the large extra dimensions can be achieved for KK models with toroidal compactification [16].

The main purpose of this paper is to construct the Lagrange function for a many-body system in the case of models with toroidal compactification. We need such a theory e.g. to calculate the formula for the advance of the periastron in the case of a binary system. The measurement of this advance for the pulsar PSR B1913+16 was performed with very high accuracy. Therefore, such measurements can be a very good test for gravitational theories. From our previous papers $[19,25,26]$ we know that gravitating bodies should have pressure/tension in the extra dimensions to satisfy the observable data for the deflection of light and the experimental restrictions for the PPN parameter $\gamma$. In this regard, the question arises of the possibility of building a many-body Lagrange function in the presence of pressure/tension in the extra dimensions. To answer this question, we need the metric's components $g_{00}$ up to $O\left(1 / c^{4}\right), g_{0 \alpha}$ up to $O\left(1 / c^{3}\right)$ and $g_{\alpha \beta}$ up to $O\left(1 / c^{2}\right)$. It is worth noting that for the expressions of the deflection of light and the PPN parameter $\gamma$, it is sufficient to calculate the metrics coefficients up to $O\left(1 / c^{2}\right)$. Obviously, the agreement with observations up to $O\left(1 / c^{2}\right)$ does not guarantee the agreement up to $O\left(1 / c^{4}\right)$. Hence, we calculate the metric's coefficients in the required orders of $1 / c$. We demonstrate that the many-body Lagrange function can be constructed for any value of $\Sigma$ where $\Sigma$ is a sum of the parameters of the equations of state in the extra dimensions. We demonstrate that the gravitational tests (PPN parameter $\gamma$, and perihelion/periastron advance) allow a very small deviation from the latent soliton value $\Sigma=-(D-3) / 2 \neq 0$. We prove that nonzero $\Sigma$ leads necessarily to the uniform smearing of the gravitating masses over the internal space. However, uniformly smeared gravitating bodies cannot have excited KK states (KK particles). As we mentioned above, KK particles were recently considered in the papers $[8,9]$. Here, the metric and form-field perturbations are studied without taking into account the reason of such fluctuations. Our present analysis clearly shows that the inclusion of the matter sources, being responsible for the perturbations, imposes strong restrictions on the model, e.g., leading to the absence of KK particles. Until now, KK particles were not detected in experiments at LHC. So, it is tempting to interpret their absence in the light of our paper (i.e. due to the smearing of the gravitating particles over the internal space). However, the absence of KK particles looks rather unnatural from the point of quantum mechanics and statistical physics (see below). Therefore, in our opinion, this is a big disadvantage of the Kaluza-Klein models with toroidal compactification.

The paper is structured as follows. In Sect. 2, we obtain the $1 / c^{2}, 1 / c^{3}$ and $1 / c^{4}$ correction terms to the metric coefficients for the considered many-body system. In Sect. 3, we demonstrate that gauge conditions lead to a uniform smearing of the gravitating bodies over the extra dimensions. The Lagrange function for the many-body system is constructed in Sect. 4. The formulas for the PPN parameters $\beta, \gamma$ and the perihelion and periastron advances are calculated in Sect. 5 . These formulas allow us to obtain experimental constraints on the parameters of the model. The main results are summarized in Sect. 6.

\section{Metric coefficients in the weak field approximation}

To construct the Lagrange function of a system of $N$ massive bodies in $(D+1)$-dimensional spacetime, we define first the nonrelativistic gravitational field created by this system. To do so, we need to get the metric coefficients in the weak field limit. The general form of the multidimensional metric is

$$
\begin{aligned}
d \mathrm{~s}^{2}= & g_{i k} d \mathrm{x}^{i} d \mathrm{x}^{k}=g_{00}\left(d \mathrm{x}^{0}\right)^{2} \\
& +2 g_{0 \mu} d \mathrm{x}^{0} d \mathrm{x}^{\mu}+g_{\mu \nu} d \mathrm{x}^{\mu} d \mathrm{x}^{\nu},
\end{aligned}
$$

where we have the Latin indices $i, k=0,1, \ldots, D$ and the Greek indices $\mu, v=1, \ldots, D$. We make the natural assumption that in the case of the absence of matter sources the spacetime is Minkowski spacetime: $g_{00}=\eta_{00}=1$, $g_{0 \mu}=\eta_{0 \mu}=0, g_{\mu \nu}=\eta_{\mu \nu}=-\delta_{\mu \nu}$. In our paper, we consider in detail the case where the extra dimensions have the topology of tori. In the presence of matter, the metric is not the Minkowskian one, and we investigate it in the weak field limit. It means that the gravitational field is weak and the velocities of the test bodies are small compared with the speed 
of light $c$. In the weak field limit the metric is only slightly perturbed from its flat spacetime value. We will define the metric (1) up to $1 / c^{2}$ correction terms. Because the coordinate $x^{0}=c t$, the metric coefficients can be expressed as follows:

$g_{00} \approx 1+h_{00}+f_{00}, \quad g_{0 \mu} \approx h_{0 \mu}+f_{0 \mu}$,

$g_{\mu \nu} \approx-\delta_{\mu \nu}+h_{\mu \nu}$,

where $h_{i k} \sim O\left(1 / c^{2}\right), f_{00} \sim O\left(1 / c^{4}\right)$ and $f_{0 \mu} \sim O\left(1 / c^{3}\right)$. In particular, $h_{00} \equiv 2 \varphi / c^{2}$ where $\varphi$ is the nonrelativistic gravitational potential. To get these correction terms, we should solve (in the corresponding orders of $1 / c$ ) the multidimensional Einstein equation

$R_{i k}=\frac{2 S_{D} \tilde{G}_{\mathcal{D}}}{c^{4}}\left(T_{i k}-\frac{1}{D-1} g_{i k} T\right)$,

where $S_{D}=2 \pi^{D / 2} / \Gamma(D / 2)$ is the total solid angle (the surface area of the $(D-1)$-dimensional sphere of the unit radius), $\tilde{G}_{\mathcal{D}}$ is the gravitational constant in the $(\mathcal{D}=D+1)$ dimensional spacetime. We consider a system of $N$ discrete massive (with rest masses $m_{p}, p=1, \ldots, N$ ) bodies. We suppose that the pressure of these bodies in the external threedimensional space is much less than their energy density. This is a natural approximation for ordinary astrophysical objects such as our Sun. For example, in GR, this approach works well for calculating the gravitational experiments in the Solar system [32]. In the case of pulsars, the pressure is not small but still much less than the energy density, and the pressureless approach was used in GR to get the formula of the periastron advance [33]. Therefore, gravitating bodies are pressureless in the external/our space. On the other hand, we suppose that they may have pressure in the extra dimensions. Therefore, the nonzero components of the energy-momentum tensor of the system can be written in the following form:

$T^{i k}=\tilde{\rho} c^{2} u^{i} u^{k}, \quad i, k=0, \ldots, 3$,

$T^{i \bar{\alpha}}=\tilde{\rho} c^{2} u^{i} u^{\bar{\alpha}}, \quad i=0, \ldots, 3 ; \bar{\alpha}=4, \ldots, D$,

$T^{\bar{\alpha} \bar{\beta}}=-p_{(\bar{\alpha}-3)} g^{\bar{\alpha} \bar{\beta}}+\tilde{\rho} c^{2} u^{\bar{\alpha}} u^{\bar{\beta}}, \quad \bar{\alpha}, \bar{\beta}=4, \ldots, D$,

where the $(D+1)$-velocity $u^{i}=d \mathrm{x}^{i} / d \mathrm{~s}$ and

$\tilde{\rho} \equiv \sum_{p=1}^{N}\left[(-1)^{D} g\right]^{-1 / 2} m_{p} \sqrt{g_{l m} \frac{d \mathrm{x}^{l}}{d \mathrm{x}^{0}} \frac{d \mathrm{x}^{m}}{d \mathrm{x}^{0}}} \delta\left(\mathbf{x}-\mathbf{x}_{p}\right)$,

where $\mathbf{x}_{p}$ is the $D$-dimensional radius vector of the $p$ th particle. In what follows, the Greek indices $\alpha, \beta=1,2,3$; $\bar{\alpha}, \bar{\beta}=4, \ldots, D$ and $\mu, v$ still run from 1 to $D$. In the extra dimensions we assume that the equations of state are

$p_{(\bar{\alpha}-3)}=\omega_{(\bar{\alpha}-3)} \tilde{\rho} c^{2}$.

If all parameters $\omega_{(\bar{\alpha}-3)}=0$, then we come back at the model considered in our paper [19]. Here, massive bodies have dust-like equations of state in all spatial dimensions. If all $\omega_{(\bar{\alpha}-3)}=-1 / 2$ (tension in the extra dimensions), then these equations of state correspond to black strings (in the case of one extra dimension, i.e. $D=4$ ) and black branes (for $D>4$ ) [28-31]. If the parameters satisfy the condition $\sum_{\bar{\alpha}} \omega_{(\bar{\alpha}-3)} \equiv \Sigma=-(D-3) / 2$, then this case corresponds to latent solitons [26]. Obviously, black strings/branes satisfy this condition.

Now, we will solve the Einstein equation (3) in the corresponding orders of $1 / c$. Obviously, for $\omega_{(\bar{\alpha}-3)}=0, \bar{\alpha}=$ $4, \ldots, D$, we should reproduce the results of the paper [19]. Because our calculations generalize the ones in [19], we skip some evident details.

First, to get the metric correction terms of the order $O\left(1 / c^{2}\right)$, the energy-momentum tensor components (4)-(6) are approximated as

$T^{00} \approx T_{00} \approx \rho c^{2}, \quad T^{\bar{\alpha} \bar{\beta}} \approx T_{\bar{\alpha} \bar{\beta}} \approx \omega_{(\bar{\alpha}-3)} \rho c^{2} \delta_{\bar{\alpha} \bar{\beta}}$,

$T^{0 \mu} \approx-T_{0 \mu} \approx 0, \quad T^{\alpha \beta} \approx T_{\alpha \beta} \approx 0$,

$T=T^{i k} g_{i k} \approx \rho c^{2}(1-\Sigma)$,

where

$\Sigma \equiv \sum_{\bar{\alpha}=4}^{D} \omega_{(\bar{\alpha}-3)}$

and we introduced the rest-mass density

$\rho(\mathbf{x})=\sum_{p=1}^{N} m_{p} \delta\left(\mathbf{x}-\mathbf{x}_{p}\right)$

Then, from the Einstein equation we get

$h_{00}=\frac{2 \varphi(\mathbf{x})}{c^{2}}, \quad h_{0 \mu}=0$,

$h_{\alpha \beta}=\frac{1-\Sigma}{D-2+\Sigma} \frac{2 \varphi(\mathbf{x})}{c^{2}} \delta_{\alpha \beta}$,

$h_{\bar{\alpha} \bar{\beta}}=\frac{\omega_{(\bar{\alpha}-3)}(D-1)+1-\Sigma}{D-2+\Sigma} \frac{2 \varphi(\mathbf{x})}{c^{2}} \delta_{\bar{\alpha} \bar{\beta}}$,

where the function $\varphi(\mathbf{x})$ satisfies the $D$-dimensional Poisson equation,

$\triangle_{D} \varphi(\mathbf{x})=2 S_{D} \tilde{G}_{\mathcal{D}} \frac{D-2+\Sigma}{D-1} \rho(\mathbf{x})$.

We recall that $\mathbf{x}$ is the $D$-dimensional radius vector. It is worth noting that if $\omega_{(\bar{\alpha}-3)}=0, \forall \bar{\alpha} \Rightarrow \Sigma=0$, then we reproduce the results of the paper [19]. On the other hand, if all $\omega_{(\bar{\alpha}-3)}=-1 / 2$, then $h_{\bar{\alpha} \bar{\beta}}=0$, and that should be the case for black strings/branes [26].

Next, we should obtain the $O\left(1 / c^{4}\right)$ and $O\left(1 / c^{3}\right)$ metric correction terms $f_{00}$ and $f_{0 \mu}$, respectively. In this case, the 
energy-momentum components read

$$
\begin{aligned}
T_{00} \approx & \rho c^{2}\left[1+\frac{\varphi}{c^{2}} \frac{3 D-4+\Sigma}{D-2+\Sigma}+\frac{v^{2}}{2 c^{2}}\right], \\
T_{0 \mu} \approx & -\rho c v^{\mu}, \\
T_{\alpha \beta} \approx & \rho v^{\alpha} v^{\beta}, \quad T_{\alpha \bar{\beta}} \approx \rho v^{\alpha} v^{\bar{\beta}}, \\
T_{\bar{\alpha} \bar{\beta}} \approx \rho c^{2}\left\{\omega _ { ( \overline { \alpha } - 3 ) } \delta _ { \overline { \alpha } \overline { \beta } } \left[1+\frac{\varphi}{c^{2}}\right.\right. & \\
& \times \frac{D-\Sigma-2\left[\omega_{(\bar{\alpha}-3)}(D-1)+1-\Sigma\right]}{D-2+\Sigma} \\
& \left.-\frac{v^{2}}{\left.2 c^{2}\right]}+\frac{v^{\bar{\alpha}} v^{\bar{\beta}}}{c^{2}}\right\},
\end{aligned}
$$

and the trace

$$
\begin{aligned}
T \approx & \rho c^{2}(1-\Sigma)+\rho \varphi \frac{(D-\Sigma)(1-\Sigma)}{D-2+\Sigma} \\
& +\rho(\Sigma-1) \frac{v^{2}}{2} .
\end{aligned}
$$

Then from the Einstein equation we get

$$
\begin{aligned}
f_{00}(\mathbf{x})= & \frac{2}{c^{4}} \varphi^{2}(\mathbf{x})+\frac{2}{c^{4}} \sum_{p} \varphi_{p}\left(\mathbf{x}-\mathbf{x}_{p}\right) \varphi^{\prime}\left(\mathbf{x}_{p}\right) \\
& +\frac{1}{c^{4}} \frac{D-\Sigma}{D-2+\Sigma} \sum_{p} \varphi_{p}\left(\mathbf{x}-\mathbf{x}_{p}\right) v_{p}^{2}
\end{aligned}
$$

and

$$
\begin{aligned}
f_{0 \mu}(\mathbf{x})= & -\frac{2}{c^{3}} \frac{D-1}{D-2+\Sigma} \sum_{p} \varphi_{p}\left(\mathbf{x}-\mathbf{x}_{p}\right) v_{p}^{\mu} \\
& -\frac{1}{c^{3}} \frac{\partial^{2} f}{\partial t \partial x^{\mu}},
\end{aligned}
$$

where the function $f$ satisfies the following equation:

$$
\triangle_{D} f=\varphi(\mathbf{x}) .
$$

\section{Gauge conditions and smearing}

It should be noted that to calculate the Ricci tensor components in the corresponding orders of $1 / c$, we use the standard gauge condition (see, e.g., Eq. (105.10) in [32])

$\partial_{k}\left(h_{i}^{k}-\frac{1}{2} h_{l}^{l} \delta_{i}^{k}\right)=0, \quad i, k=0,1, \ldots, D$,

where $h_{i}^{k} \equiv \eta^{k m} h_{m i}$. Hence,

$h_{0}^{0}=\eta^{00} h_{00}=h_{00}, \quad h_{\nu}^{\mu}=\eta^{\mu \kappa} h_{\nu \kappa}=-h_{\mu \nu}$.
Therefore,

$h_{0}^{0}=\frac{2 \varphi(\mathbf{x})}{c^{2}}, \quad h_{\alpha}^{\beta}=-\frac{1-\Sigma}{D-2+\Sigma} \frac{2 \varphi(\mathbf{x})}{c^{2}} \delta_{\alpha}^{\beta}$,

$h_{\bar{\alpha}}^{\bar{\beta}}=-\frac{\omega_{(\bar{\alpha}-3)}(D-1)+1-\Sigma}{D-2+\Sigma} \frac{2 \varphi(\mathbf{x})}{c^{2}} \delta_{\bar{\alpha}}^{\bar{\beta}}$,

$h_{l}^{l}=\frac{2(\Sigma-1)}{D-2+\Sigma} \frac{2 \varphi(\mathbf{x})}{c^{2}}$.

Let us check that these solutions satisfy the condition (24). For $i=0$, we get immediately

$\partial_{k}\left(h_{0}^{k}-\frac{1}{2} h_{l}^{l} \delta_{0}^{k}\right)=\partial_{0}\left(h_{0}^{0}-\frac{1}{2} h_{l}^{l}\right)=0+O\left(\frac{1}{c^{3}}\right)$.

For $i=\beta$ we have

$$
\begin{aligned}
& \partial_{k}\left(h_{\beta}^{k}-\frac{1}{2} h_{l}^{l} \delta_{\beta}^{k}\right)=\partial_{\alpha}\left(h_{\beta}^{\alpha}-\frac{1}{2} h_{l}^{l} \delta_{\beta}^{\alpha}\right) \\
& \quad=\left[-\frac{1-\Sigma}{D-2+\Sigma}+\frac{1-\Sigma}{D-2+\Sigma}\right] \frac{2}{c^{2}} \partial_{\beta} \varphi=0,
\end{aligned}
$$

that is, the condition is automatically satisfied. For $i=\bar{\beta}$ we obtain

$$
\begin{aligned}
\partial_{k}\left(h_{\bar{\beta}}^{k}-\frac{1}{2} h_{l}^{l} \delta_{\bar{\beta}}^{k}\right) & =\partial_{\bar{\alpha}}\left(h_{\bar{\beta}}^{\bar{\alpha}}-\frac{1}{2} h_{l}^{l} \delta_{\bar{\beta}}^{\bar{\alpha}}\right) \\
& =-\frac{\omega_{(\bar{\beta}-3)}(D-1)}{D-2+\Sigma} \frac{2}{c^{2}} \partial_{\bar{\beta}} \varphi=0 .
\end{aligned}
$$

In order to satisfy this condition, we should demand either $\omega_{(\bar{\beta}-3)}=0$ or $\partial_{\bar{\beta}} \varphi=0$. Because we consider the general case $\omega_{(\bar{\beta}-3)} \neq 0$, we must choose the latter condition. Moreover, the gravitational tests require nonzero $\omega_{(\bar{\beta}-3)}$ (see Sect. $5)$. Therefore, the presence of nonzero pressure/tension in the extra dimensions results in metric coefficients which do not depend on the coordinates of the internal space, i.e. the gravitating masses should be uniformly smeared over the extra dimensions. In this case, the rest-mass density (11) should be rewritten in the form $\rho(\mathbf{x}) \rightarrow \rho(\mathbf{r})=\sum_{p} m_{p} \delta(\mathbf{r}-$ $\left.\mathbf{r}_{p}\right) / \prod_{\bar{\alpha}} a_{(\bar{\alpha}-3)}$, where $\mathbf{r}_{p}$ is the three-dimensional radius vector of the $p$ th particle in the external space, $a_{(\bar{\alpha}-3)}$ are the periods of the tori (i.e. $\prod_{\bar{\alpha}} a_{(\bar{\alpha}-3)}$ is the volume of the internal space). Then Eq. (15) is reduced to the ordinary three-dimensional Poisson equation

$\triangle_{3} \varphi(\mathbf{r})=4 \pi G_{N} \sum_{p} m_{p} \delta\left(\mathbf{r}-\mathbf{r}_{p}\right)$

with the solution

$\varphi(\mathbf{r})=-\sum_{p} \frac{G_{N} m_{p}}{\left|\mathbf{r}-\mathbf{r}_{p}\right|}=\sum_{p} \varphi_{p}\left(\mathbf{r}-\mathbf{r}_{p}\right)$, 
where $G_{N}$ is the Newtonian gravitational constant:

$4 \pi G_{N}=\frac{2 S_{D}(D-2+\Sigma)}{(D-1) \prod_{\bar{\alpha}} a_{(\bar{\alpha}-3)}} \tilde{G}_{\mathcal{D}}$.

Hereafter, $\mathbf{r}, \mathbf{r}_{p}$ are radius vectors in three-dimensional external space.

In the case of the smearing, Eq. (23) has the following solution:

$f(\mathbf{r})=-\frac{G_{N}}{2} \sum_{p} m_{p}\left|\mathbf{r}-\mathbf{r}_{p}\right|$,

where we used the well-known equation $\triangle_{3} r=2 / r$ in the three-dimensional flat space. Because

$$
\begin{aligned}
& \frac{\partial}{\partial t}\left(\frac{\partial\left|\mathbf{r}-\mathbf{r}_{p}\right|}{\partial x^{\alpha}}\right)=\frac{\partial}{\partial t}\left(\frac{x^{\alpha}-x_{p}^{\alpha}}{\left|\mathbf{r}-\mathbf{r}_{p}\right|}\right)=\frac{1}{\left|\mathbf{r}-\mathbf{r}_{p}\right|^{2}} \\
& \quad \times\left[-v_{p}^{\alpha}\left|\mathbf{r}-\mathbf{r}_{p}\right|-\frac{x^{\alpha}-x_{p}^{\alpha}}{\left|\mathbf{r}-\mathbf{r}_{p}\right|} \sum_{\beta}\left(x^{\beta}-x_{p}^{\beta}\right)\left(-v_{p}^{\beta}\right)\right],
\end{aligned}
$$

we get for $f_{0 \alpha}$ :

$f_{0 \alpha}=\frac{G_{N}}{2 c^{3}} \sum_{p} \frac{m_{p}}{\left|\mathbf{r}-\mathbf{r}_{p}\right|}\left(\frac{3 D-2-\Sigma}{D-2+\Sigma} v_{p}^{\alpha}+n_{p}^{\alpha}\left(\mathbf{n}_{p} \mathbf{v}_{p}\right)\right)$,

where we introduce the three-dimensional unit vector in the direction from the $p$ th particle to a point with the radius vector $\mathbf{r}$ :

$n_{p}^{\alpha}=\frac{x^{\alpha}-x_{p}^{\alpha}}{\left|\mathbf{r}-\mathbf{r}_{p}\right|}$

and $\left(\mathbf{n}_{p} \mathbf{v}_{p}\right)=\sum_{\beta} n_{p}^{\beta} v_{p}^{\beta}$.

It should be noted that, to get the formula (22), we used the following gauge condition:

$\frac{\partial f_{0}^{\mu}}{\partial x^{\mu}}-\frac{1}{2} \frac{\partial h_{\mu}^{\mu}}{\partial x^{0}}=0$,

where $f_{0}^{\mu}=\eta^{k \mu} f_{0 k}=-f_{0 \mu}$. In the case of smearing, this condition is reduced to

$\frac{\partial f_{0}^{\beta}}{\partial x^{\beta}}-\frac{1}{2} \frac{\partial h_{\mu}^{\mu}}{\partial x^{0}}=0$,

where we recall that $\alpha, \beta=1,2,3$ and $\mu, v=1, \ldots, D$. Taking into account the following auxiliary equations:

$\frac{\partial}{\partial x^{\beta}} \frac{1}{\left|\mathbf{r}-\mathbf{r}_{p}\right|}=-\frac{n_{p}^{\beta}}{\left|\mathbf{r}-\mathbf{r}_{p}\right|^{2}}$,

$\sum_{\beta} \frac{\partial}{\partial x^{\beta}}\left(n_{p}^{\beta} \frac{\left(\mathbf{n}_{p} \mathbf{v}_{p}\right)}{\left|\mathbf{r}-\mathbf{r}_{p}\right|}\right)=\frac{\left(\mathbf{n}_{p} \mathbf{v}_{p}\right)}{\left|\mathbf{r}-\mathbf{r}_{p}\right|^{2}}$,

$\frac{\partial}{\partial t} \frac{1}{\left|\mathbf{r}-\mathbf{r}_{p}\right|}=\frac{\left(\mathbf{n}_{p} \mathbf{v}_{p}\right)}{\left|\mathbf{r}-\mathbf{r}_{p}\right|^{2}}$, we can easily see that the condition (40) is satisfied:

$$
\begin{aligned}
\frac{\partial f_{0}^{\beta}}{\partial x^{\beta}}- & \frac{1}{2} \frac{\partial h_{\mu}^{\mu}}{\partial x^{0}} \\
= & \frac{G_{N}}{2 c^{3}}\left\{\frac{3 D-2-\Sigma}{D-2+\Sigma} \sum_{p} m_{p} \frac{\left(\mathbf{n}_{p} \mathbf{v}_{p}\right)}{\left|\mathbf{r}-\mathbf{r}_{p}\right|^{2}}-\sum_{p} m_{p} \frac{\left(\mathbf{n}_{p} \mathbf{v}_{p}\right)}{\left|\mathbf{r}-\mathbf{r}_{p}\right|^{2}}\right. \\
& \left.-\frac{2(D-\Sigma)}{D-2+\Sigma} \sum_{p} m_{p} \frac{\left(\mathbf{n}_{p} \mathbf{v}_{p}\right)}{\left|\mathbf{r}-\mathbf{r}_{p}\right|^{2}}\right\}=0 .
\end{aligned}
$$

Because the presence of pressure/tension in the extra dimensions requires the uniform smearing of the gravitating masses over the internal space, we provide the metric coefficients in this case:

$$
\begin{aligned}
g_{00} \approx & +\frac{2 \varphi(\mathbf{r})}{c^{2}}+\frac{2 \varphi^{2}(\mathbf{r})}{c^{4}} \\
& +\frac{2 G_{N}^{2}}{c^{4}} \sum_{p} \frac{m_{p}}{\left|\mathbf{r}-\mathbf{r}_{p}\right|} \sum_{q \neq p} \frac{m_{q}}{\left|\mathbf{r}_{p}-\mathbf{r}_{q}\right|} \\
& -\frac{D-\Sigma}{D-2+\Sigma} \frac{G_{N}}{c^{4}} \sum_{p} \frac{m_{p} v_{p}^{2}}{\left|\mathbf{r}-\mathbf{r}_{p}\right|},
\end{aligned}
$$

$g_{0 \alpha} \approx \frac{3 D-2-\Sigma}{D-2+\Sigma} \frac{G_{N}}{2 c^{3}} \sum_{p} \frac{m_{p}}{\left|\mathbf{r}-\mathbf{r}_{p}\right|} v_{p}^{\alpha}$

$$
+\frac{G_{N}}{2 c^{3}} \sum_{p} \frac{m_{p}}{\left|\mathbf{r}-\mathbf{r}_{p}\right|} n_{p}^{\alpha}\left(\mathbf{n}_{p} \mathbf{v}_{p}\right),
$$

$g_{\alpha \beta} \approx\left(-1+\frac{1-\Sigma}{D-2+\Sigma} \frac{2 \varphi(\mathbf{r})}{c^{2}}\right) \delta_{\alpha \beta}$,

$g_{\bar{\alpha} \bar{\beta}} \approx\left(-1+\frac{\omega_{(\bar{\alpha}-3)}(D-1)+1-\Sigma}{D-2+\Sigma} \frac{2 \varphi(\mathbf{r})}{c^{2}}\right) \delta_{\bar{\alpha} \bar{\beta}}$,

where the potential $\varphi(\mathbf{r})$ is given by (33).

Therefore we have shown, in this section, that, to be compatible with the gravitational tests, the gravitating masses should be uniformly smeared over the internal space. This conclusion has the following important effect. Suppose that we have solved for the considered particle the multidimensional quantum Schrödinger equation and found its wave function $\Psi(\mathbf{x})$. In general, this function depends on all spatial coordinates $\mathbf{x}=(\mathbf{r}, \mathbf{y})$, where $\mathbf{y}$ are the coordinates in the internal space, and we can expand it in appropriate eigenfunctions of the compact internal space, i.e. in the KaluzaKlein modes. The ground state corresponds to the absence of these particles. In this state the wave function may depend only on the coordinates $\mathbf{r}$ of the external space. The classical rest-mass density is proportional to the probability density $|\Psi|^{2}$. Therefore, the requirement that the rest-mass density depends only on the coordinates of the external space means that the particle can be only in the ground quantum state, and KK excitations are absent. This looks very unnatural from the point of view of quantum and statistical physics, because a nonzero temperature must result in excitations. 


\section{Lagrange function for a many-body system}

Let us construct now the Lagrange function of the manybody system described above. To perform it, we will follow the procedure described in [32] (see §106). The Lagrange function of a particle $p$ with mass $m_{p}$ in the gravitational field created by the other bodies is given by the expression

$$
\begin{aligned}
L_{p} & =-m_{p} c \frac{d \mathrm{~s}_{p}}{d t} \\
& =-m_{p} c^{2}\left(g_{00}+2 \sum_{\mu} g_{0 \mu} \frac{v_{p}^{\mu}}{c}+\sum_{\mu \nu} g_{\mu \nu} \frac{v_{p}^{\mu} v_{p}^{\nu}}{c^{2}}\right)^{1 / 2},
\end{aligned}
$$

where the metric coefficients are taken at $\mathbf{r}=\mathbf{r}_{p}$. We should keep in mind that in the case of smeared gravitating masses (smeared over the extra dimensions), the components of the velocity in the extra dimensions are equal to zero: $v_{p}^{\mu}=$ $\left(v_{p}^{\alpha}, v_{p}^{\bar{\alpha}}\right)=\left(v_{p}^{\alpha}, 0\right)$. It is convenient to rewrite the metric coefficients (45)-(47) in the following form:

$$
\begin{aligned}
& g_{00} \approx 1+\frac{1}{c^{2}} \gamma_{00}^{(1)}+\frac{1}{c^{4}} \gamma_{00}^{(2)}, \quad g_{0 \alpha} \approx \frac{1}{c^{3}} \gamma_{0 \alpha}, \\
& g_{\alpha \beta} \approx\left(-1+\frac{1}{c^{2}} \gamma_{(\alpha)}\right) \delta_{\alpha \beta},
\end{aligned}
$$

where the meaning of the functions $\gamma$ is evident. Then, we get

$$
\begin{aligned}
\frac{d \mathrm{~s}_{p}}{d t} \approx & c\left\{1+\frac{1}{2 c^{2}}\left[\gamma_{00}^{(1)}-v_{p}^{2}\right]\right. \\
& +\frac{1}{2 c^{4}}\left[\gamma_{00}^{(2)}+2 \sum_{\alpha} \gamma_{0 \alpha} v_{p}^{\alpha}+\sum_{\alpha \beta} \gamma_{(\alpha)} \delta_{\alpha \beta} v_{p}^{\alpha} v_{p}^{\beta}\right] \\
& \left.-\frac{1}{8 c^{4}}\left[\gamma_{00}^{(1)}-v_{p}^{2}\right]^{2}\right\} .
\end{aligned}
$$

Substituting the explicit form of the metric coefficients (45)-(47), we obtain

$$
\begin{aligned}
L_{p}= & -m_{p} c^{2}+\frac{m_{p} v_{p}^{2}}{2}+\frac{m_{p} v_{p}^{4}}{8 c^{2}}+G_{N} \sum_{s} \frac{m_{p} m_{s}}{\left|\mathbf{r}-\mathbf{r}_{s}\right|} \\
& -\frac{1}{2 c^{2}} G_{N}^{2} \sum_{s} \sum_{q} \frac{m_{p} m_{s} m_{q}}{\left|\mathbf{r}-\mathbf{r}_{s}\right|\left|\mathbf{r}-\mathbf{r}_{q}\right|} \\
& -\frac{1}{c^{2}} G_{N}^{2} \sum_{s} \sum_{q \neq s} \frac{m_{p} m_{s} m_{q}}{\left|\mathbf{r}-\mathbf{r}_{s}\right|\left|\mathbf{r}_{s}-\mathbf{r}_{q}\right|} \\
& +\frac{1}{2 c^{2}} G_{N} \sum_{S} \frac{m_{p} m_{s}}{\left|\mathbf{r}-\mathbf{r}_{s}\right|}\left[\mathfrak{a}(D, \Sigma) v_{s}^{2}+2\left(\mathfrak{b}(D, \Sigma)+\frac{1}{2}\right) v_{p}^{2}\right. \\
& \left.-\mathfrak{c}(D, \Sigma)\left(\mathbf{v}_{S} \mathbf{v}_{p}\right)-\left(\mathbf{n}_{S} \mathbf{v}_{S}\right)\left(\mathbf{n}_{S} \mathbf{v}_{p}\right)\right]
\end{aligned}
$$

Here, we use the following abbreviations:

$$
\begin{aligned}
\mathfrak{a}(D, \Sigma) & \equiv \frac{D-\Sigma}{D-2+\Sigma}, \quad \mathfrak{b}(D, \Sigma) \equiv \frac{1-\Sigma}{D-2+\Sigma}, \\
\mathfrak{c}(D, \Sigma) & \equiv \frac{3 D-2-\Sigma}{D-2+\Sigma} .
\end{aligned}
$$

We recall that in the expression (51) $\mathbf{r}=\mathbf{r}_{p}$ and all infinite terms should be cast out. For our purposes, it is sufficient to consider the case of two particles. Then, for particle ' 1 ', we have the following expression:

$$
\begin{aligned}
L_{1}= & \mathrm{f}\left(\mathbf{v}_{1}^{2}\right)+G_{N} \frac{m_{1} m_{2}}{\left|\mathbf{r}-\mathbf{r}_{2}\right|}-\frac{1}{2 c^{2}} G_{N}^{2} \frac{m_{1} m_{2}^{2}}{\left|\mathbf{r}-\mathbf{r}_{2}\right|^{2}} \\
& -\frac{1}{c^{2}} G_{N}^{2} \frac{m_{1}^{2} m_{2}}{\left|\mathbf{r}-\mathbf{r}_{2}\right|\left|\mathbf{r}_{1}-\mathbf{r}_{2}\right|} \\
& +\frac{1}{2 c^{2}} \frac{G_{N} m_{1} m_{2}}{\left|\mathbf{r}-\mathbf{r}_{2}\right|}\left[\mathfrak{a}(D, \Sigma) v_{2}^{2}+2\left(\mathfrak{b}(D, \Sigma)+\frac{1}{2}\right) v_{1}^{2}\right. \\
& \left.-\mathfrak{c}(D, \Sigma)\left(\mathbf{v}_{1} \mathbf{v}_{2}\right)-\left(\mathbf{n}_{2} \mathbf{v}_{2}\right)\left(\mathbf{n}_{2} \mathbf{v}_{1}\right)\right]
\end{aligned}
$$

where $\mathrm{f}\left(\mathbf{v}_{1}^{2}\right)=m_{1} v_{1}^{2} / 2+m_{1} v_{1}^{4} /\left(8 c^{2}\right)$ and we drop the term $-m_{1} c^{2}$.

The total Lagrange function of the two-body system should be constructed so that it leads to the correct values of the forces $\partial L_{p} /\left.\partial \mathbf{r}\right|_{\mathbf{r}=\mathbf{r}_{p}}$ acting on each of the bodies for a given motion of the others [32]. To achieve it, we first will differentiate $L_{1}$ with respect to $\mathbf{r}$, setting $\mathbf{r}=\mathbf{r}_{1}$ afterwards. Then we should integrate this expression with respect to $\mathbf{r}_{1}$. Following this prescription and taking into account the useful auxiliary relation

$$
\begin{gathered}
\left.\left(-\frac{1}{2 c^{2}} G_{N}^{2} m_{1} m_{2}^{2} \frac{\partial}{\partial \mathbf{r}} \frac{1}{\left|\mathbf{r}-\mathbf{r}_{2}\right|^{2}}-\frac{G_{N}^{2}}{c^{2}} \frac{m_{1}^{2} m_{2}}{\left|\mathbf{r}_{1}-\mathbf{r}_{2}\right|} \frac{\partial}{\partial \mathbf{r}} \frac{1}{\left|\mathbf{r}-\mathbf{r}_{2}\right|}\right)\right|_{\mathbf{r}=\mathbf{r}_{1}} \\
=-\frac{1}{2 c^{2}} G_{N}^{2} m_{1} m_{2}\left(m_{1}+m_{2}\right) \frac{\partial}{\partial \mathbf{r}_{1}} \frac{1}{\left|\mathbf{r}_{1}-\mathbf{r}_{2}\right|^{2}}
\end{gathered}
$$

we obtain from (53) the two-body Lagrange function

$$
\begin{aligned}
L_{1}^{(2)}= & \tilde{\mathrm{f}}\left(\mathbf{v}_{1}^{2}, \mathbf{v}_{2}^{2}\right)+\frac{G_{N} m_{1} m_{2}}{r_{12}}-\frac{G_{N}^{2} m_{1} m_{2}\left(m_{1}+m_{2}\right)}{2 c^{2} r_{12}^{2}} \\
& +\frac{G_{N} m_{1} m_{2}}{2 c^{2} r_{12}}\left[\mathfrak{a}(D, \Sigma) v_{2}^{2}+(2 \mathfrak{b}(D, \Sigma)+1) v_{1}^{2}\right. \\
& \left.-\mathfrak{c}(D, \Sigma)\left(\mathbf{v}_{1} \mathbf{v}_{2}\right)-\left(\mathbf{n}_{12} \mathbf{v}_{1}\right)\left(\mathbf{n}_{12} \mathbf{v}_{2}\right)\right]
\end{aligned}
$$

where $\tilde{\mathrm{f}}\left(\mathbf{v}_{1}^{2}, \mathbf{v}_{2}^{2}\right)=\sum_{a=1}^{2} m_{a} v_{a}^{2} / 2+\sum_{a=1}^{2} m_{a} v_{a}^{4} /\left(8 c^{2}\right)$ and $r_{12} \equiv\left|\mathbf{r}_{1}-\mathbf{r}_{2}\right|$. It can be easily seen that $\partial L_{1} /\left.\partial \mathbf{r}\right|_{\mathbf{r}=\mathbf{r}_{1}}=$ $\partial L_{1}^{(2)} / \partial \mathbf{r}_{1}$. In the same way we can construct the two-body Lagrange function $L_{2}^{(2)}$ from the Lagrange function $L_{2}$ for particle ' 2 ': 


$$
\begin{aligned}
L_{2}^{(2)}= & \tilde{\mathrm{f}}\left(\mathbf{v}_{1}^{2}, \mathbf{v}_{2}^{2}\right)+\frac{G_{N} m_{1} m_{2}}{r_{12}}-\frac{G_{N}^{2} m_{1} m_{2}\left(m_{1}+m_{2}\right)}{2 c^{2} r_{12}^{2}} \\
& +\frac{G_{N} m_{1} m_{2}}{2 c^{2} r_{12}}\left[\mathfrak{a}(D, \Sigma) v_{1}^{2}+(2 \mathfrak{b}(D, \Sigma)+1) v_{2}^{2}\right. \\
& \left.-\mathfrak{c}(D, \Sigma)\left(\mathbf{v}_{1} \mathbf{v}_{2}\right)-\left(\mathbf{n}_{12} \mathbf{v}_{1}\right)\left(\mathbf{n}_{12} \mathbf{v}_{2}\right)\right] .
\end{aligned}
$$

It is worth noting that both $L_{1}^{(2)}$ and $L_{2}^{(2)}$ are reduced to the Lagrange function of the two-body system in [32] if we assume that $D=3, \Sigma=0$.

Obviously, the Lagrange functions $L_{1}^{(2)}$ and $L_{2}^{(2)}$ should be symmetric with respect to permutations of particles 1 and 2 and should coincide with each other. This requires the following condition:

$\mathfrak{a}(D, \Sigma)=2 \mathfrak{b}(D, \Sigma)+1$,

which is satisfied identically for any value of $\Sigma$. Therefore, we construct the two-body Lagrange function for any value of the parameters of the equation of state in the extra dimensions.

\section{Gravitational tests}

It can easily be seen that the components of the metric in the external/our space (45)-(47) as well as the two-body Lagrange functions (55) and (56) exactly coincide with the corresponding expressions in General Relativity for the value $\Sigma=\sum_{\bar{\alpha}} \omega_{(\bar{\alpha}-3)}=-(D-3) / 2$, i.e. the latent soliton case [26]. Black strings and black branes are particular cases of it. Therefore, the known gravitational tests in this case give the same results as for GR. In other words, we get good agreement with observations. It is interesting to obtain an experimental restriction on deviation from this value. For this purpose, we write $\Sigma$ in the following form:

$\Sigma=-\frac{D-3}{2}+\varepsilon$

and find the experimental limitations on $\varepsilon$.

\section{$P P N$ parameters}

To get the parameterized post-Newtonian parameters (PPN) $\beta$ and $\gamma$, we consider the case of one particle at rest. Then we can easily obtain from Eqs. (45) and (47)

$\beta=1, \quad \gamma=\frac{1-\Sigma}{D-2+\Sigma}$,

i.e. the PPN parameter $\beta$ exactly coincides with the value in the GR. There are strong experimental restrictions on the value of $\gamma$. The tightest constraint on $\gamma$ comes from the Shapiro time-delay experiment using the Cassini spacecraft, namely $\gamma-1=(2.1 \pm 2.3) \times 10^{-5}$ [33-35]. In our case

$\gamma-1 \approx-\frac{4 \varepsilon}{D-1}$.
Therefore, the Shapiro time-delay experiment results in the following limitation:

$|\varepsilon| \lesssim \frac{D-1}{2} \times 10^{-5}$.

\section{Perihelion shift of Mercury}

For a test body orbiting around the gravitating mass $m$, the perihelion shift for one period is given by the formula $[33,36]$

$$
\begin{aligned}
\delta \psi & =\frac{1}{3}(2+2 \gamma-\beta) \frac{6 \pi G_{N} m}{c^{2} a\left(1-e^{2}\right)} \\
& \equiv \frac{1}{3}(2+2 \gamma-\beta) \delta \psi_{\mathrm{GR}},
\end{aligned}
$$

with $a$ and $e$ being the semi-major axis and the eccentricity of the ellipse, respectively. $\delta \psi_{\mathrm{GR}}$ is the value for GR. In the case of Mercury this calculated value is equal to 42.98 arcsec per century $[33,37]$. This predicted relativistic advance agrees with the observations to about $0.1 \%$ [33]. Substituting the PPN parameters (59) in this formula, we obtain the advance in our case:

$\delta \psi=\frac{1}{3} \frac{D-\Sigma}{D-2+\Sigma} \delta \psi_{\mathrm{GR}} \approx\left(1-\frac{8}{3(D-1)} \varepsilon\right) \delta \psi_{\mathrm{GR}}$.

Obviously, to be in agreement with the observation in a way not worse than GR, the parameter $\varepsilon$ should satisfy the condition

$|\varepsilon| \lesssim \frac{3(D-1)}{8} \times 10^{-3}$.

Therefore, this limitation is less strong than (61).

Periastron shift of the relativistic binary pulsar PSR B1913+16

A much stronger limitation can be found from the measurement of the periastron shift of the relativistic binary pulsar. First, the advance of periastron in these systems is many orders of magnitude bigger than for the Mercury. Second, the measurements are extremely accurate. For example, for the pulsar PSR B1913+16 the shift is $4.226598 \pm 0.000005$ degree per year [38]. For such systems the pulsar and companion have comparable masses. In the case of GR, the solution for the orbital parameters yields mass estimates for the pulsar and its companion, $m_{1}=1.4398 \pm 0.0002 M_{\odot}$ and $m_{2}=1.3886 \pm 0.0002 M_{\odot}$, respectively. It is worth noting that these are calculated values (not observable!) which are valid for GR. Because two bodies have comparable masses (and one of them cannot be considered as a test body), to get a formula for the advance we need a two-body Lagrangian. Then, following problem 3 in $\$ 106$ [32] we get for our twobody Lagrangians (55) and (56) the desired formula in the form of (63) with the well-known GR expression

$\delta \psi_{\mathrm{GR}}=\frac{6 \pi G_{N}\left(m_{1}+m_{2}\right)}{c^{2} a\left(1-e^{2}\right)}$. 
In future, independent measurements of masses $m_{1}$ and $m_{2}$ will allow us to obtain a restriction of high accuracy on the parameter $\varepsilon$.

\section{Summary}

In this paper, we have constructed the Lagrange function for a two-body system in the case of Kaluza-Klein models with toroidal compactification of the extra dimensions. The case of more than two bodies is straightforward. We supposed that gravitating bodies are pressureless in the external space. This is a natural approximation for ordinary astrophysical objects such as the Sun. For example, this approach works well for calculating the gravitational experiments in the Solar system [32]. In the case of pulsars, the pressure is not small but still much less than the energy density. Hence, the pressureless approach is used in General Relativity to get the formula (65) which is in very good agreement with the observations of the advance of the periastron of the pulsar PSR B1913+16.

With respect to the internal space, we supposed that gravitating masses may have nonzero parameters $\omega_{(\bar{\alpha}-3)}(\bar{\alpha}=$ $4, \ldots, D)$ of the equations of state in the extra dimensions. We have shown that the Lagrange function of this many-body system can be constructed for any value of the parameter $\Sigma=\sum_{\bar{\alpha}} \omega_{(\bar{\alpha}-3)}$.

To construct the many-body Lagrangian, as well as to get the formulas for the gravitational tests, we obtained the metric's components $g_{00}$ up to $O\left(1 / c^{4}\right), g_{0 \alpha}$ up to $O\left(1 / c^{3}\right)$, and $g_{\alpha \beta}$ up to $O\left(1 / c^{2}\right)$. These expressions exactly coincide with the corresponding formulas in GR for the value $\Sigma=\sum_{\bar{\alpha}} \omega_{(\bar{\alpha}-3)}=-(D-3) / 2$. This is the latent soliton case [26]. Black strings/branes are particular cases of it with all $\omega_{(\bar{\alpha}-3)}=-1 / 2 \forall \bar{\alpha}$. Obviously, the known gravitational tests (PPN parameters, perihelion/periastron shift) in this case give the same results as for GR. On the other hand, we used these tests to get the restrictions on the deviation from the latent soliton value. At present, the strongest restriction follows from the time delay of radar echoes (the Cassini spacecraft mission). The two-body Lagrange function allowed us to get the formula for the advance of the periastron. In future, when the masses of the binary pulsar system PSR B1913+16 will be measured (rather than calculated using the formula of GR), the advance of this periastron can be used to get the restriction with very high accuracy. All obtained limitations indicate a very small deviation from the latent soliton value. Therefore, the pressureless case $\Sigma=0$ in the internal space is forbidden, in full agreement with the results of the paper [19]. This conclusion does not depend on the size of the extra dimensions. The physical reason of it is that in the case of toroidal compactification, only in the case of latent solitons the variations of the total volume of the internal space are absent [27].
One more important result obtained in this paper is worth noting. As we have shown above [25-27], the tension in the internal spaces is the necessary condition to satisfy the gravitational experiments in KK models with toroidal compactification. In our paper, we have proven that the presence of pressure/tension in the internal space necessarily leads to the uniform smearing of the gravitating masses over the internal space. For example, black strings/branes have tension in the internal space (see, e.g., [39]). Therefore, they should be smeared. However, uniformly smeared gravitating bodies cannot have excited KK states (KK particles), which looks unnatural from the point of view of quantum mechanics and statistical physics. In our opinion, this is a big disadvantage of the Kaluza-Klein models with toroidal compactification. It is of interest to check this property for models with other types of compactification (e.g. Ricci-flat, spherical). This is the subject of our subsequent study.

Acknowledgments This work was supported in part by the "Cosmomicro-physics-2" programme of the Physics and Astronomy Division of the National Academy of Sciences of Ukraine. The work of M. Eingorn was supported by NSF CREST award HRD-0833184 and NASA grant NNX09AV07A.

Open Access This article is distributed under the terms of the Creative Commons Attribution License which permits any use, distribution, and reproduction in any medium, provided the original author(s) and the source are credited.

Funded by $\mathrm{SCOAP}^{3}$ / License Version CC BY 4.0.

\section{References}

1. Th. Kaluza, Zum Unitätsproblem der Physik, Sitzungsber. d. Preuss. Akad. d. Wiss. 966 (1921)

2. O. Klein, Quantentheorie und fünfdimensionale Relativitätstheorie. Zeitschrift für Physik 37, 895 (1926)

3. J. Polchinski, String Theory, Superstring Theory and Beyond, vol. 2. (Cambridge University Press, Cambridge, 1998)

4. G. Bhattacharyya, A. Datta, S.K. Majee, A. Raychaudhuri, Nucl. Phys. B 821, 48 (2009). [arXiv:0904.0937 [hep-ph]]

5. A. Salam, J. Strathdee, On Kaluza-Klein theory. Ann. Phys. 141, 316 (1982)

6. P. van Nieuwenhuizen, The complete mass spectrum of $D=11$ supergravity compactified on $S(4)$ and a general mass formula for arbitrary cosets M(4). Class. Quant. Gravity 2, 1 (1985)

7. H. Kim, L. Romans, P. van Nieuwenhuizen, The mass spectrum of chiral $N=2 D=10$ supergravity on S(5). Phys. Rev. D 32, 389 (1985)

8. K. Hinterbichler, J. Levin, C. Zukowski, Kaluza-Klein towers on general manifolds (2013). [arXiv:1310.6353 [hep-th]]

9. A.R. Brown, A. Dahlen, Stability and spectrum of compactifications on product manifolds (2013). [arXiv:1310.6360 [hep-th]]

10. N. Arkani-Hamed, S. Dimopoulos, G. Dvali, Phys. Lett. B 429, 263 (1998). [arXiv:hep-ph/9803315]

11. N. Arkani-Hamed, S. Dimopoulos, G. Dvali, Phys. Rev. D 59, 086004 (1999). [arXiv:hep-ph/9807344]

12. A. Kehagias, K. Sfetsos, Phys. Lett. B 472, 39 (2000). [arXiv: hep-ph/9905417]

13. A.O. Barvinsky, S.N. Solodukhin, Nucl. Phys. B 675, 159 (2003). [arXiv:hep-th/0307011] 
14. P. Callin, C.P. Burgess, Nucl. Phys. B 752, 60 (2006). [arXiv: hep-ph/0511216]

15. M. Eingorn, A. Zhuk, Phys. Rev. D 80, 124037 (2009). [arXiv: 0907.5371 [hep-th]]

16. M. Eingorn, A. Zhuk, Class. Quant. Gravity 27, 055002 (2010). [arXiv:0910.3507 [gr-qc]]

17. C.D. Hoyle et al., Phys. Rev. D 70, 042004 (2004). [arXiv:hep-ph/ 0405262]

18. P. Xu, Y. Ma, Phys. Lett. B 656, 165 (2007). [arXiv:0710.3677 [gr-qc]]

19. M. Eingorn, A. Zhuk, Class. Quant. Gravity 27, 205014 (2010). [arXiv: 1003.5690 [gr-qc]]

20. I. Antoniadis, N. Arkani-Hamed, S. Dimopoulos, G. Dvali, Phys. Lett. B 436, 257 (1998). [arXiv:hep-ph/9804398]

21. G.F. Giudice, R. Rattazzi, J.D. Wells, Nucl. Phys. B 544, 3 (1999). [arXiv:hep-ph/9811291]

22. T. Han, J.D. Lykken, R.-J. Zhang, Phys. Rev. D 59, 105006 (1999). [arXiv:hep-ph/9811350]

23. J. Lykken, S. Nandi, Phys. Lett. B 485, 224 (2000). [arXiv:hep-ph/ 9908505]

24. S. Hannestad, G. Raffelt, Phys. Rev. Lett. 87, 051301 (2001). [arXiv:hep-ph/0103201]

25. M. Eingorn, A. Zhuk, Phys. Rev. D 83, 044005 (2011). [arXiv: 1010.5740 [qr-qc]]

26. M. Eingorn, O. de Medeiros, L. Crispino, A. Zhuk, Phys. Rev. D 84, 024031 (2011). [arXiv:1101.3910 [gr-qc]]

27. M. Eingorn, A. Zhuk, Phys. Lett. B 713, 154 (2012). [arXiv:1201.1756 [gr-qc]]
28. J. Traschen, D. Fox, Class. Quant. Gravity 21, 289 (2004). [arXiv:gr-qc/0103106]

29. P.K. Townsend, M. Zamaklar, Class. Quant. Gravity 18, 5269 (2001). [arXiv:hep-th/0107228]

30. T. Harmark, N.A. Obers, JHEP 0405, 043 (2004). [arXiv:hep-th/ 0403103]

31. D. Kastor, J. Traschen, JHEP 0609, 022-039 (2006). [arXiv:hep-th/ 0607051]

32. L.D. Landau, E.M. Lifshitz, in The Classical Theory of Fields, 4 edn, vol. 2. Course of Theoretical Physics Series (Pergamon Press, Oxford, 2000)

33. C.M. Will, Was Einstein Right? Testing Relativity at the Centenary, ed. by A. Ashtekar. 100 Years of Relativity: Space-time Structure: Einstein and Beyond (World Scientific, Singapore, 2005). [arXiv: gr-qc/0504086]

34. Bh. Jain, J. Khoury, Tests of Gravity (2010). [arXiv:1004.3294 [astro-ph]]

35. B. Bertotti, L. Iess, P. Tortora, Nature 425, 374 (2003)

36. C.M. Will, Theory and Experiment in Gravitational Physics (Cambridge University Press, Cambridge, 2000)

37. A.M. Nobili, C.M. Will, Nature 320, 39 (1986)

38. J.M. Weisberg, D.J. Nice, J.H. Taylor, Astrophys. J. 722, 1030 (2010). [arXiv:1011.0718 [astro-ph]]

39. D. Kastor, J. Traschen, JHEP 0609, 022 (2006). [arXiv:hep-th/ 0607051] 